\title{
WINNING RATE IN THE FULL-INFORMATION BEST-CHOICE PROBLEM
}

\author{
ALEXANDER V. GNEDIN, ${ }^{*}$ Utrecht University \\ DENIS I. MIRETSKIY, ${ }^{* *}$ University of Twente
}

\begin{abstract}
Following a long-standing suggestion by Gilbert and Mosteller, we derive an explicit formula for the asymptotic winning rate in the full-information best-choice problem.

Keywords: Best-choice problem; Poisson embedding

2000 Mathematics Subject Classification: Primary 60G40

Secondary $60 \mathrm{G} 70$
\end{abstract}

\section{Introduction}

Let $X_{1}, X_{2} \ldots$ be a sequence of independent uniform $[0,1]$ random variables. The fullinformation best-choice problem, as introduced by Gilbert and Mosteller [3], asks us to find a stopping rule $\tau_{n}$ to maximise the probability

$$
P_{n}(\tau):=\mathrm{P}\left(X_{\tau}=\max \left\{X_{1}, \ldots, X_{n}\right\}\right)
$$

over all stopping rules $\tau \leq n$ adapted to the sequence $\left(X_{i}\right)$. The name 'full-information' was attached to the problem to stress that the observer learns the exact values of the $X_{i}$ s and knows their distribution, in contrast to the 'no-information' problem where only the relative ranks of observations are available (see [15] for a survey and history of the best-choice or 'secretary' problems). Because the stopping criterion (1) depends only on ranks of the observations, the instance of uniform distribution covers, in fact, the general case of sampling from arbitrary continuous distribution.

Gilbert and Mosteller [3] showed that the optimal stopping rule is of the form

$$
\tau_{n}=\min \left\{i: X_{i}=\max \left\{X_{1}, \ldots, X_{i}\right\} \text { and } X_{i} \geq d_{n-i}\right\}
$$

where $d_{k}$ is a sequence of decision numbers defined by the equation

$$
\sum_{j=1}^{k} \frac{d_{k}^{-j}-1}{j}=1 \quad \text { for } k \geq 1 \text { and } d_{0}=0 .
$$

Received 12 February 2007.

* Postal address: Department of Mathematics, Utrecht University, Postbus 80010, 3508 TA Utrecht, The Netherlands. Email address: gnedin@math.uu.nl

** Postal address: Department of Applied Mathematics, University of Twente, Postbus 217, 7500 AE Enschede, The Netherlands. Email address: d.miretskiy@math.utwente.nl 
Gilbert and Mosteller also proved that $d_{k} \uparrow 1$ in such a way that $k\left(1-d_{k}\right) \rightarrow c$ for $c=0.804 \ldots$, where $c$ is the solution to the transcendental equation

$$
\int_{0}^{c} x^{-1}\left(\mathrm{e}^{x}-1\right) \mathrm{d} x=1,
$$

and they provided numerical evidence that the best-choice optimal probability $P_{n}^{*}:=P_{n}\left(\tau_{n}\right)$ converges to a limit $P^{*}=0.580164 \ldots$. The limiting value was justified by different methods in subsequent work (see [1], [4], [14], [15]) along with the explicit formula

$$
P^{*}=\mathrm{e}^{-c}+\left(\mathrm{e}^{c}-c-1\right) \int_{1}^{\infty} \mathrm{e}^{-c x} x^{-1} \mathrm{~d} x
$$

(see [14]).

Refinements and generalisations of the results of [3] appeared in [4], [5], [6], [9], [10], [11], and [13]. Still, one interesting feature of the optimal stopping rule seems to have not been discussed in the literature. We refer to the tiny Section 3e in [3] where Gilbert and Mosteller say

One would correctly anticipate that as $n$ increases, the probability of winning at a given draw tends to zero. On the other hand, $n \mathrm{P}$ (win at draw $i$ ) tends to a constant for $i / n$ tending to a constant $\lambda$.

Spelled out in detail, Gilbert and Mosteller claimed existence of the limit

$$
w(t)=\lim _{i, n \rightarrow \infty, i / n \rightarrow t} n \mathrm{P}\left(\tau_{n}=i, X_{i}=\max \left\{X_{1}, \ldots, X_{n}\right\}\right),
$$

where $t \in[0,1]$ stands for their $\lambda$. Such a function may be called the asymptotic winning rate since it tells us how the chance of correctly recognising the maximum is distributed over time; hence, the best-choice total probability must satisfy

$$
P^{*}=\int_{0}^{1} w(t) \mathrm{d} t .
$$

In this paper, we prove the conjecture of [3] regarding the convergence and we derive an explicit formula for the winning rate (4). In fact, we show more: the function $w$ appears as the exact winning rate in a continuous-time version of the best-choice problem associated with a planar Poisson process (as developed in [4], [5], [7], and [16]).

\section{The Poisson framework}

We start by recalling the setup from [4] and [5]. Consider a homogeneous planar Poisson process (PPP) in the semi-infinite strip $R=[0,1] \times]-\infty, 0]$, with Lebesgue measure as intensity. The generic atom $a=(t, x) \in R$ of the PPP is understood as score $x$ observed at time $t$. Let $\mathcal{F}=\left(\mathcal{F}_{t}, t \in[0,1]\right)$ be the filtration with $\mathcal{F}_{t}$ being the $\sigma$-algebra generated by the PPP restricted to $[0, t] \times]-\infty, 0]$. We say that an atom $a=(t, x)$ of the PPP is a record if there are no other PPP-atoms northwest of $a$. The maximum of the PPP is an atom $a^{*}=\left(t^{*}, x^{*}\right)$ with the largest $x$-value. Alternatively, the maximum $a^{*}$ can be defined as the last record of the PPP, that is the record with the largest $t$-value. For $\tau$, i.e. a $\mathcal{F}$-adapted stopping rule with values in $[0,1]$, the performance of $\tau^{*}$ is defined as the probability of the event $\left\{\tau=t^{*}\right\}$, interpreted 
as the best choice from the PPP. The associated best-choice problem amounts to maximising the probability of the event $\left\{\tau=t^{*}\right\}$.

In a Poissonised version of the Gilbert-Mosteller problem, the observations sampled from the $[0,1]$ uniform distribution arrive on $[0, \ell]$ at epochs of a rate 1 Poisson process [1], [2], [8], [12]. This is equivalent to the PPP setup with background space $[0, \ell] \times[0,1]$, which can be mapped linearly onto $[0,1] \times[-\ell, 0]$ so that the componentwise order of points is preserved. Now, the optimal stopping in $[0,1] \times[-\ell, 0]$ fits in the framework with the background space $R$ by a minor modification of the stopping criterion: a stopping rule $\tau$ adapted to $\mathcal{F}$ is evaluated by the probability of the event $\left\{\tau=t^{*}, a^{*}>-\ell\right\}$ that stopping occurs at the maximum atom and above $-\ell$. In this sense we shall speak of a constrained best-choice problem.

Let $\Gamma=\{(t, x) \in R:-x(1-t)<c\}$, where $c$ is as in (3). It is known [4] that the optimal stopping rule is the first time (if any) when the record process enters $\Gamma$, that is

$$
\tau^{*}=\min \{t: \text { there is a record } a=(t, x) \in \Gamma\}
$$

(or $\tau^{*}=1$ if no such $t \in[0,1$ [ exists). Similarly, the optimal stopping rule for the constrained problem is the first time (if any) when the record process enters $\Gamma(\ell):=\Gamma \cap([0,1] \times[0,-\ell])$.

Let

$$
g(\ell, t):=\mathrm{P}\left(\tau^{*}=t^{*}, t^{*}<t, x^{*}>-\ell\right)
$$

be the probability that $\tau^{*}$ wins by stopping above $-\ell$ and before $t$, and let

$$
g(\infty, t):=\mathrm{P}\left(\tau^{*}=t^{*}, t^{*}<t\right) .
$$

By the above relation, between the constrained and unconstrained problems, we have

$$
g(\ell, t)=g(\infty, t) \quad \text { for } 0 \leq t \leq\left(1-\frac{c}{\ell}\right)_{+} .
$$

The winning rate in the Poisson problem is defined as

$$
w(t)=\partial_{t} g(\infty, t) .
$$

\section{Computing the rate}

Because the atoms southwest of $(x,-\ell)$ fall outside the stopping region $\Gamma(\ell)$, we have $\partial_{\ell} g(\ell, t)=0$ and $g(\infty, t)=g(c /(1-t), t)$, for $\ell>c /(1-t)$. To determine $\partial_{\ell} g(\ell, t)$ for $\ell>c /(1-t)$, consider two rectangles $R_{1}=[0, t] \times[-\ell, 0]$ and $R_{2}=[0, t] \times[-\ell+\delta, 0]$, with small $\delta>0$. The optimal constrained stopping rules in $R_{1}$ and $R_{2}$ stop before $t$ at distinct atoms if and only if the record process enters $\Gamma(\ell)$ at some atom $a_{0}=(\sigma, \xi) \in$ $\left[(1-c / \ell)_{+}, t\right] \times[-\ell,-\ell+\delta]$. Now, stopping at $a_{0} \in R_{1} \backslash R_{2}$ is a win if $a_{0}=a^{*}$, which occurs with probability

$$
p_{1}=\mathrm{e}^{-\ell} \ell\left(t-\left(1-\frac{c}{\ell}\right)_{+}\right) \frac{\delta}{\ell}+o(\delta)=\mathrm{e}^{-\ell}\left(t-\left(1-\frac{c}{\ell}\right)_{+}\right) \delta+o(\delta) .
$$

On the other hand, stopping in $R_{2}$ is a win (and stopping at $a_{0}$ is a loss) if $a_{0}$ is followed by some $k>0$ atoms in $[\sigma, 1] \times[-\ell, 0]$, the leftmost of these $k$ atoms appears within $[\sigma, t] \times[-\ell, 0]$ and it is the overall maximum $a^{*}$ which is an event of probability

$$
p_{2}=\mathrm{e}^{-\ell} \sum_{k=1}^{\infty} \frac{c^{k+1}}{(k+1) !}\left[1-(k+1) \frac{t-(1-c / \ell)_{+}}{c / \ell} \frac{(1-t)^{k}}{(c / \ell)^{k}}-\frac{(1-t)^{k+1}}{(c / \ell)^{k+1}}\right] \frac{1}{k} \frac{\delta}{\ell}+o(\delta) .
$$




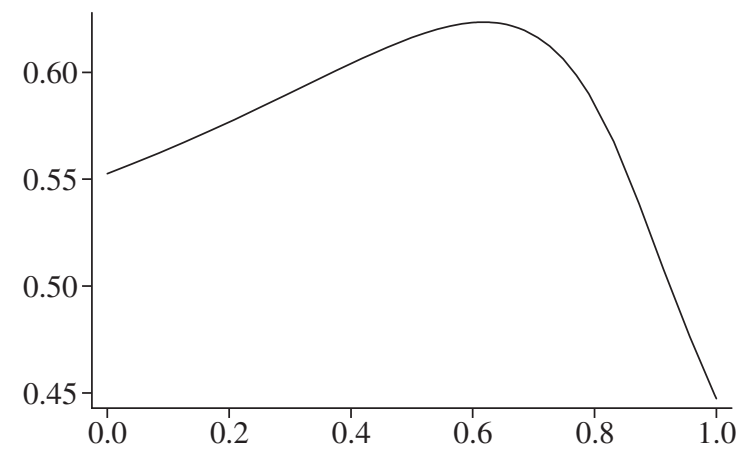

Figure 1: The winning rate. Reprinted with permission from the Journal of the American Statistical Association. Copyright 1966 by the American Statistical Association. All rights reserved.

It follows that

$$
\partial_{\ell} g(\ell, t)=\lim _{\delta \rightarrow 0} \frac{p_{1}-p_{2}}{\delta}=\mathrm{e}^{-\ell} \int_{(1-c / \ell)_{+}}^{t}\left(1-\sum_{k=1}^{\infty}\left[\frac{\ell^{k}(1-\sigma)^{k}}{k ! k}-\frac{\ell^{k}(1-t)^{k}}{k ! k}\right]\right) \mathrm{d} \sigma .
$$

Now, computing the mixed second derivative $\partial_{\ell t} g(\ell, t)$ and integrating in $\ell$ from 0 to $c /(1-t)$, we obtain the winning rate in the Poisson problem, which is our main result.

Proposition 1. The winning rate is given by the formula

$$
w(t)=-\mathrm{e}^{-c}+\frac{\mathrm{e}^{-c t}-\mathrm{e}^{-c t /(1-t)}}{t}+\frac{\mathrm{e}^{-c t}-t \mathrm{e}^{-c}}{1-t}+\frac{c}{1-t}\left\{I\left(\frac{c}{1-t}, c\right)-I\left(\frac{c t}{1-t}, c t\right)\right\},
$$

where $c$ is as in (3) and, for $0<s<t$,

$$
I(t, s)=\int_{s}^{t} \xi^{-1} \mathrm{e}^{-\xi} \mathrm{d} \xi
$$

The boundary values of $w$ are $w(0)=1-\mathrm{e}^{-c}=0.5526 \ldots$ and $w(1)=\mathrm{e}^{-c}=0.4473 \ldots$, in accordance with [3, Figure 3]. The graph of (5) in Figure 1 exhibits a curve identical to that in [3, Figure 3].

The special value (3) of $c$ was not used in the argument, hence the right-hand side of (5) gives the winning rate for every stopping rule defined by a stopping region like $\Gamma$ but with an arbitrary positive constant in place of $c$. We also note that the winning rate in the constrained problem coincides with $w(t)$ for $t<(1-c / \ell)_{+}$.

\section{Embedding and convergence}

It remains to show that $w$ given by (5) is indeed the limiting value for the finite- $n$ problem in (4). To that end, we will exploit the embedding technique from [4].

With $n$ fixed, divide $R$ in strips $J_{i}=[(i-1) / n, i / n[\times]-\infty, 0], \quad i=1, \ldots, n$. Consider a sequence $y_{n}=\left(\left(T_{i}, Y_{i}\right), i=1, \ldots, n\right)$, where $\left(T_{i}, Y_{i}\right)$ is an atom with the largest $x$-component within the strip $J_{i}$. Observe that the point process of records in $y_{n}$ is a subset of the set of records of the PPP in $R$; in particular, $\max \left\{Y_{i}\right\}=x^{*}$. By homogeneity of the 
PPP we have $T_{1}, Y_{1}, \ldots, T_{n}, Y_{n}$ jointly independent, with each $T_{i}$ uniformly distributed on $\left[(i-1) / n, i / n\right.$ [ and each $Y_{i}$ exponentially distributed on ] $\left.-\infty, 0\right]$ with rate $1 / n$. It follows that the discrete time optimal stopping problem of recognising the maximum in $y_{n}$ is equivalent to the Gilbert-Mosteller problem with exponentially distributed observations.

Let $\hat{\tau}_{n}$ be the optimal stopping rule for recognising the maximum in $\mathcal{y}_{n}$. We shall view $\hat{\tau}_{n}$ as a strategy for choosing the maximum of PPP with the additional option of partial return meaning that $\hat{\tau}_{n}$ assumes values in $[0,1]$, that

$$
\left\{\frac{i-1}{n}<\hat{\tau}_{n} \leq \frac{i}{n}\right\} \in \mathcal{F}_{i / n},
$$

and that $\left\{(i-1) / n<\hat{\tau}_{n} \leq i / n\right\}$ is associated with the stopping at $\left(T_{i}, Y_{i}\right)$. Explicitly, $\hat{\tau}_{n}$ stops at the first time the sequence of $y_{n}$-records enters

$$
\left.\left.\Gamma_{n}=\bigcup_{i=1}^{n}\right] \frac{i-1}{n}, \frac{i}{n}\right] \times\left[b_{n-i}, 0\right]
$$

where $b_{k}=n \log d_{k}$ and the $d_{k}$ s are the decision numbers as in (2). The partial return option implies that the winning chance of $\hat{\tau}_{n}$ is higher than that of $\tau^{*}$.

Let $a^{\prime}$ be the last record before $a^{*}$. We can easily check that $\tau^{*}$ and $\hat{\tau}$ may differ only if either $a^{*}$ or $a^{\prime}$ hit the domain

$$
\Delta_{n}:=\left(\Gamma_{n} \backslash \Gamma\right) \cup\left(\Gamma \backslash \Gamma_{n}\right) .
$$

By [4, Equation (11)] we have $\left((i-1) / n, b_{n-i}\right) \notin \Gamma$ and $\left(i / n, b_{n-i}\right) \in \Gamma$, for $i=1, \ldots, n$. This combined with the fact that the distribution of $t^{*}$ is uniform and that of $x^{*}$ is exponential yields

$$
n \mathrm{P}\left(a^{*} \in \Delta_{n} \cap J_{i}\right)<\exp \left(\frac{-n c}{n-i+1}\right)-\exp \left(\frac{-n c}{n-i}\right)=O\left(n^{-1}\right)
$$

uniformly in $i \leq n$. A similar estimate holds also for $a^{\prime}$ and, because

$$
\mathrm{P}\left(\frac{i-1}{n}<\hat{\tau}_{n} \leq \frac{i}{n}\right)=\mathrm{P}\left(\tau_{n}=i\right), \quad w\left(\frac{i}{n}\right)=\mathrm{P}\left(\tau^{*}=t^{*}, a^{*} \in J_{i}\right)+O\left(n^{-1}\right)
$$

(the second statement holds since $w$ is smooth on $[0,1]$ ), we can deduce the following result.

Proposition 2. As $n \rightarrow \infty$ the optimal stopping rule $\tau_{n}$ satisfies

$$
\max _{1 \leq i \leq n}\left\{\left|w\left(\frac{i}{n}\right)-n \mathrm{P}\left(\tau_{n}=i, X_{i}=\max \left\{X_{1}, \ldots, X_{n}\right\}\right)\right|\right\}=O\left(n^{-1}\right)
$$

where $w$ is given by (5).

\section{References}

[1] Berezovsky, B. A. And Gnedin, A. V. (1984). The Best Choice Problem. Nauka, Moscow.

[2] BoJdECKI, T. (1977). On optimal stopping of independent random variables appearing to a renewal process with random time horizon. Bol. Soc. Mat. Mexicana 22, 35-40.

[3] Gilbert, J. P. And Mosteller, F. (1966). Recognizing the maximum of a sequence. J. Amer. Statist. Assoc. 61, $35-73$.

[4] Gnedin, A. V. (1996). On the full-information best-choice problem. J. Appl. Prob. 33, 678-687. 
[5] Gnedin, A. V. (2004). Best choice from the planar Poisson process. Stoch. Process. Appl. 111, 317-354.

[6] Gnedin, A. V. (2005). Objectives in the best-choice problems. Sequential Anal. 24, 177-188.

[7] Gnedin, A. V. (2007). Recognising the last record of a sequence. Stochastics 79, 199-209.

[8] Gnedin, A. V. AND SaKaguchi, M. (1992). On a best choice problem related to the Poisson process. Contemp. Math. 125, 59-64.

[9] Hill, T. ANd Kennedy, D. (1992). Sharp inequalities for optimal stopping with rewards based on ranks. Ann. Appl. Prob. 2, 503-517.

[10] Petruccelli, J. D. (1980). On a best choice problem with partial information. Ann. Statist. 8, 1171-1174.

[11] PorosińsKi, Z. (1987). The full-information best choice problem with a random number of observations. Stoch. Process. Appl. 24, 293-307.

[12] SaKaguchi, M. (1976). Optimal stopping problems for randomly arriving offers. Math. Japon. 21, $210-217$.

[13] Samuel-Cahn, E. (1996). Optimal stopping with random horizon with application to the full-information best-choice problem with random freeze. J. Amer. Statist. Assoc. 91, 357-364.

[14] Samuels, S. M. (1982). Exact solutions for the full information best choice problem. Mimeo Ser. 82-17, Department of Statistics, Purdue University.

[15] Samuels, S. M. (1991). Secretary problems. In Handbook of Sequential Analysis, eds B. K. Ghosh and P. K. Sen, Marcel Dekker, New York, pp. 381-405.

[16] Samuels, S. M. (2004). Why do these quite different best-choice problems have the same solutions? Adv. Appl. Prob. 36, 398-416. 